\title{
Splinting duration for replanted avulsed teeth
}

\author{
Abstracted from \\ Hinckfuss SE, Messer LB. \\ Splinting duration and periodontal outcomes for replanted avulsed teeth: \\ a systematic review. Dent Traumatol 2009; 25: 150-157 \\ Address for correspondence: Professor Louise Brearley Messer, School of Dental Science, \\ Faculty of Medicine, Dentistry and Health Sciences, University of Melbourne, \\ 720 Swanston Street, Victoria 3010, Australia. \\ E-mail: ljbm@unimelb.edu.au
}

\section{Question: For how long should re-implanted avulsed teeth be splinted?}

Data sources Four databases were searched for relevant citations: Ovid Medline, Cochrane Library, PubMed and ISI Web of Science. Study selection Studies were included in the review if they had been conducted on humans and addressed the management of traumatised permanent teeth. Only English language papers were considered. Final selection was based on relevance to the question, and availability of sufficient detail in the paper.

Data extraction and synthesis A qualitative summary of the studies identified is presented.

Results The review was limited by the studies' small sample sizes, variability in study design methodology and observation periods, and lack of uniformity in terminology for outcomes. A total of 138 replanted avulsed permanent teeth could be pooled from four papers, each reporting both short-term splinting ( 14 days or less) and long-term splinting (over 14 days) in accord with current clinical guidelines. Conclusions The evidence appears inconclusive for any association between short-term splinting and increased likelihood of functional periodontal healing, acceptable healing, or decreased development of replacement resorption. The study found no evidence to contraindicate the current guidelines and suggests that the likelihood of successful periodontal healing after replantation is unaffected by splinting duration. Pending future research to the contrary, it is recommended that dentists continue to use the currently-recommended splinting periods when replanting avulsed permanent teeth.

\section{Commentary}

The aim of this systematic review of the literature was to identify studies where splinting periods of both $<14$ days and $>14$ days had been used following avulsion, and thus appraise the evidence for current guidance on splinting times for avulsed permanent teeth - most recommendations are for a period of 7-14 days. Not surprisingly, given the tightness of the inclusion criteria, the initial 36470 citations were swiftly whittled down to just four studies. The authors state that an additional 10 studies could have been included if a little more detail had been available from the paper. Unfortunately, contacting the authors of the papers for the additional information met with little success: four stated the data were, "in storage, and unavailable"; four did not reply; and two could not be contacted. The review authors are not the first to find retrieving additional detail problematic. With regard to systematic reviews, the regrettable fact is not just that there are so few data out there, but that so much of what is out there is of so little use. Systematic reviews have an important role in highlighting both what type of studies are needed to answer the question posed, and what information should be provided to answer that question, and the Editors of journals, present and future, and their teams of referees are key people in ensuring that all relevant data are provided in publications.

The review authors had little choice but to pool the data from the four studies, while acknowledging the wide variations in study designs and outcome measures. This gave a total of 136 teeth, 66 of which were splinted for $<14$ days, and 72 were splinted for $>14$ days. Although this appears to be a relatively even distribution, one centre splinted the majority of their sample (83\%) for a short time ( $<14$ days), another had a nearly 50:50 split, whereas the remaining two both splinted the majority of their sample (76\%) for the longer period of time ( $>14$ days).

Why the differences between the centres? And within the centres, why were they sometimes splinting for longer, and sometimes for shorter times? Was there random allocation between the two time periods? Was it just happenstance? Or were clinicians using their clinical judgement to assess which teeth needed longer splinting times to achieve a good outcome? The reason is important in evaluating the information, but is not given in this paper. What is of interest is that when splinting for the recommended period (7-14 days), two centres reported success rates of $75 \%$ or higher, whereas two reported success rates of $27 \%$ or lower, which raises the question of what the two more successful centres were doing differently from the others.

The authors are to be commended on a systematic review which is thorough and well-reported, such that it could be repeated by another group with little difficulty. The conclusion, that splinting duration does not seem to be a significant factor in the success or otherwise of replantations, is useful.

\section{Practice points \\ Avulsed permanent teeth should be splinted for 7-14 days following replantation. \\ - Should a clinician feel a tooth should be splinted for a little longer (eg, because of excessive mobility, or suspected alveolar fracture), this should not affect the probability of periodontal healing.}

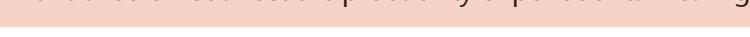

Dafydd Evans

Preventive and Children's Section, Dundee Dental Hospital and School, Park Place, Dundee, Scotland, UK

Evidence-Based Dentistry (2009) 10, 104. doi:10.1038/sj.ebd.6400680 